\title{
HANDOFF ORDERING USING LINK QUALITY ESTIMATOR FOR MULTIMEDIA COMMUNICATIONS IN WIRELESS NETWORKS
}

\author{
Tsung-Nan Lin, Po-Chiang Lin \\ Graduate Institute of Communication Engineering \\ National Taiwan University \\ Email: tsungnan@ ntu.edu.tw,r92942075@ntu.edu.tw
}

\begin{abstract}
Traditional handoff ordering methods adopt the received signal strength (RSS) as the basis of prioritization. However, the RSS is not the only one metric to represent the user perceived quality of service, since many other factors, like the packet length, interference, and the modulation/codec schemes, would also affect it. In this paper, we propose a handoff ordering method based on packet success rate (PSR) for multimedia communications in wireless networks. The priority of a handoff request is based on its current PSR, the PSR degradation rate, and the minimum PSR requirement of its service class. The major contribution of our method is that we improve the user perceived QoS during the handoff process. Simulation results indicate that our method can effectively improve the handoff call dropping probability with little or no increase of the new call blocking probability.
\end{abstract}

\section{INTRODUCTION}

Channel is a valuable and limited resource for wireless communications. In wireless cellular communications, the number of channels in a base station (BS) determines the number of mobile units (MU) that can be served simultaneously by this base station. When the total channels of a BS are all allocated to the calls, the new call arising from this BS would be blocked, and the handoff call from the neighboring BS would be either dropped or put into the handoff request queue to wait for available channel. For the latter case, a suitable ordering method is required to prioritize the handoff requests. Since blocking a new call, from a user's perspective, is much more acceptable than dropping a proceeding call during the handoff process, the main objective of the handoff ordering methods is to reduce the handoff call dropping probability.

There are some works about the handoff ordering issue. Tekinay et al. proposed a measurement-based prioritization scheme (MBPS) which prioritizes the handoff requests in the queue based on the received signal strength (RSS) [1]. A handoff request with a lower RSS would be assigned a higher priority since it is more likely to be dropped soon due to bad signal quality. What MBPS does not take into consideration is that each MU travels with different speed and direction. The RSS of the MU with fast speed leaving from its serving BS would be degraded faster than that with slow speed. Ebersman et al. proposed a signal prediction priority queuing (SPPQ) method which predicts each MU's expected remaining time $t_{L}$ to reach the minimum RSS requirement [2]. The prediction is

\footnotetext{
This work was supported in part by YULON NISSAN Motor Co under grant 94-S-C04-H and by Taiwan National Science Council under grant 94-2219-E-002-017.
}

based on the MU's current RSS and the RSS degradation rate, which takes the different speed of the MU into consideration. The MU with the smallest value of $t_{L}$ would be handed off first.

The above two methods, MBPS and SPPQ, are designed for the wireless communication networks with only one service class. For wireless communication networks nowadays, transmitting multimedia information over wireless networks is gaining more and more attention all over the world. That is there would be more than one service class coexisting in the same wireless communication network, and all service classes have different connection durations, bandwidth requirements, and link quality requirements. Hence it is important that the network provides quality-of-service (QoS) guarantees. It requires careful consideration to handle the handoff ordering issue in this circumstance. Chang et al. propose a handoff ordering method called signal strength for multimedia communications (SSMC) [3]. In SSMC, the priority of a handoff request is calculated using the following three values: a static priority value, the RSS, and the RSS degradation rate. A higher value of the static priority is assigned to the more important service class. However, the large differences between the static priority values would prevent the less important calls from gaining access until all the more important calls in the queue are served. Thus not all of the handoff requests with different service classes get a fair competition. Besides, the adopted RSS can not directly represent the user perceived QoS. The perceived quality of multimedia services is not affected by RSS only. Many other factors, like the packet length, interference, and the modulation/codec schemes, would also affect the perceived quality of multimedia services. For example, the MU with high RSS may not get good QoS when it suffers serious interference. Besides, not all multimedia applications have the same QoS requirements. Each multimedia application has its specific QoS requirement. For example, the real-time video and voice applications require high transmission quality since the bad quality of these applications would not be accepted by human being. On the contrary, non-real-time data services like Webbrowsing and E-Mail do not require QoS since the erroneous packets can be retransmitted by the automatic repeat request (ARQ) mechanisms. Since RSS is a measurement in physical layer, it cannot be directly or easily mapped to the QoS of the upper layers. Therefore, a new handoff ordering metric other than RSS is necessary.

In this paper, we use the packet success rate (PSR) which is the average number of successfully received packets as the link quality estimator to determine the handoff priority. PSR is suitable for the handoff ordering metric in wireless multimedia communications because it can be directly or easily mapped to the QoS requirement of the multimedia application. Given a 
specific QoS requirement, it is easy to know the corresponding PSR requirement. While PSR can really represent the absolute perceived QoS of the handoff requests in the queue, RSS can only represent the relative comparison of them in physical layer. Hence it is more suitable to use PSR as the handoff ordering metric. When the PSR is below the minimum PSR requirement of the service class, the user would feel unsatisfied with the service quality, and the connection would be terminated by the user, even when the RSS is still acceptable.

Recently several works use the metric (PSR) to design in various wireless applications. D. Couto [9] used the metric to find the high-throughput paths in a multi-hop routing algorithm in wireless networks. Lal et al. [4] show that PSR can be measured in an energy-efficient way. They discovered "reasonably few channel measurements are sufficient to obtain a good estimate of the cost metric".

Therefore, we are motivated to propose a handoff ordering method, quality prediction priority queuing (QPPQ), which uses PSR to predict the remaining time to reach the minimum PSR requirement. By $\mathrm{QPPQ}$, the priority of a handoff request is based on its current PSR, the PSR degradation rate, and the minimum PSR requirement of its service class. Our objective is to reduce the handoff call dropping probability for all multimedia service classes. From simulation results, we show that the performance of QPPQ performs better than the previous works [1-3].

\section{QUALITY PREDICTION PRIORITY QUEUING}

\section{A. Design Principle}

In order to reduce the handoff call dropping probability, most of the previous works about the handoff ordering problem [1-3] calculate the priorities of the handoff requests based on the RSS and follow either one or both of the following two criteria.

1) The MU with worse quality requires an earlier handoff than the MU with better quality.

2) The MU with faster speed requires an earlier handoff than the MU with slower speed.

Traditional methods adopt RSS as the quality metric. Now we analyze the previously proposed SSMC method [3] by the following example to illustrate that some problems will happen even when these two criteria are obeyed, thus the new handoff ordering method is necessary. In SSMC, the priority of the handoff request $\mathrm{j}$ is calculated as

$$
P(j)=p_{j} \times \Delta R S S(j) \times \frac{1}{R S S(j)}
$$

where $p_{j}$ is the static priority value for the service class of $j$ which depending on how important the service class is. 1/RSS(j) is the reciprocal of its RSS which considers to represent the transmission quality of the first criterion. $\Delta \mathrm{RSS}(\mathrm{j})$ is the RSS degradation rate which estimates the speed of the MU in the second criterion.

Assume that $\mathrm{MU}_{\mathrm{A}}$ and $\mathrm{MU}_{\mathrm{B}}$ locate at different distances from the same BS $\left(\mathrm{MU}_{\mathrm{A}}\right.$ is nearer to the $\mathrm{BS}$ than $\mathrm{MU}_{\mathrm{B}}$ is) and are leaving the BS with the same speed $\mathrm{V}$ and direction, as Fig. 1 shows. We also assume that these two MUs belong to the same service class, thus their static priority values in Eq. (1) are the same. As these two MUs move, their received RSS will be degraded. Eventually, at point X, the calls will be dropped due to the unacceptable RSS. Since they move at the same speed, because of the first criterion, $\mathrm{MU}_{\mathrm{B}}$ should be assigned a higher priority since it will reach the point $\mathrm{X}$ sooner than $\mathrm{MU}_{\mathrm{A}}$.

Considering the simple free space propagation model [5], the RSS is given by the Friis free space equation,

$\operatorname{RSS}(d)(d B)=10 \times \log \left(\frac{P_{t} G_{t} G_{r} \lambda^{2}}{(4 \pi)^{2} d^{2} L}\right)$

where $P_{t}$ is the transmitted power, $G_{t}$ and $G_{r}$ are the transmitter and receiver antenna gains, $\lambda$ is the wavelength in meters, $d$ is the transmitter-receiver separation distance in meters, and $\mathrm{L}$ is the system loss factor. Without losing generality, we use the parameters shown in Table 1 and combine (1) and (2) to find the relationship between the handoff priority $\mathrm{P}(\mathrm{j})$ and the separation distance $\boldsymbol{d}$ between the serving BS and the MV, as Fig. 2 shows. Contrary to the intuition that $\mathrm{MU}_{\mathrm{B}}$ should be assigned a higher priority, we find out that the priority $P(j)$ is a monotonic decreasing function of the distance $d$. As the distance $d$ increases, the corresponding priority $\mathrm{P}(\mathrm{j})$ decreases. Since $\mathrm{MU}_{\mathrm{A}}$ is located nearer to the $\mathrm{BS}$ than $\mathrm{MU}_{\mathrm{B}}$ is, it means that $\mathrm{MU}_{\mathrm{A}}$ would be assigned a higher priority that does not follow the first criterion mentioned above. This is because that although $\mathrm{MU}_{\mathrm{A}}$ and $\mathrm{MU}_{\mathrm{B}}$ move at the same speed, the RSS degradation rate of $M U_{A}$ is larger than that of $\mathrm{MU}_{\mathrm{B}}$ due to the nonlinear property between RSS and the distance d described in (2). Thus, when considering $\triangle \mathrm{RSS}, \mathrm{MU}_{\mathrm{A}}$ should get higher priority while considering 1/RSS(j), $\mathrm{MU}_{\mathrm{B}}$ should get higher priority. Simply multiply $\triangle$ RSS with $1 / \operatorname{RSS}(\mathrm{j})$ would cause problems. This will result in the increase of handoff call dropping probability when serving the multimedia services in wireless networks. Furthermore, Eq. (1) does not consider the situation when the MU is stopped because of the traffic light or traffic jam. When the MU is not moving, the priority becomes the lowest $(\triangle \mathrm{RSS}=0)$ and the call may be dropped eventually.

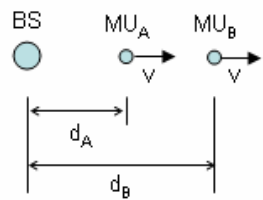

Fig. 1. A simple scenario for case 1.

\begin{tabular}{|c|c|c|c|c|c|c|c|}
\hline $\mathrm{P}_{\mathrm{t}}$ & $\mathrm{G}_{\mathrm{t}}$ & $\mathrm{G}_{\mathrm{r}}$ & $\lambda$ & $\mathrm{L}$ & $\mathrm{V}$ & $\begin{array}{c}\text { sample } \\
\text { interval }\end{array}$ & $\mathrm{p}_{\mathrm{j}}$ \\
\hline $20 \mathrm{~W}$ & 1 & 1 & $0.33 \mathrm{~m}$ & 1 & $\begin{array}{c}60 \\
\mathrm{~km} / \mathrm{hr}\end{array}$ & $1 \mathrm{sec}$. & 1 \\
\hline
\end{tabular}

Table 1. The example parameters to be used in (1) and (2).

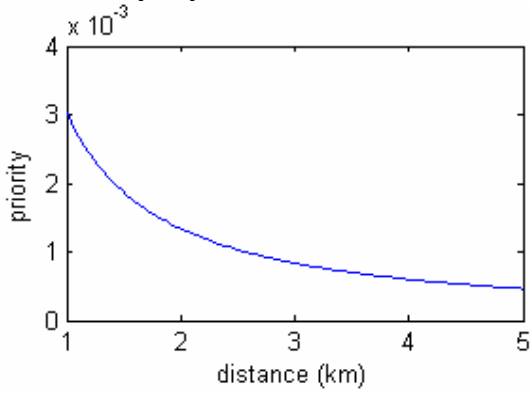

Fig. 2. The relationship between the handoff ordering priority $P(j)$ and the BS-MU distance d.

The illustrative example shows the problem of using RSS as the quality estimation by tradition methods [1-3] to determine 
the handoff priority. Furthermore, RSS can not really represent the user perceived QoS. It is just a measurement in physical layer and cannot be directly or easily mapped to the QoS of the upper layers. Many other factors, like the packet length, interference, and the modulation/codec schemes, would also affect the perceived quality of multimedia services. For example, the MU with high RSS may not get good QoS when it suffers serious interference.

PSR is suitable for the handoff ordering metric in wireless multimedia communications because it can be directly or easily mapped to the QoS requirement of the multimedia application. When the perceived QoS of the MU is below its minimum QoS requirement, the QoS is not acceptable and the call would be terminated by the user, even when it has not reached the minimum RSS requirement. Since each MU leaving from its serving BS will reach the minimum PSR requirement at some future time, we denote the remaining time to reach the minimum PSR requirement as $T$, as the main mechanism to determine the handoff priority. The handoff request with smaller $\mathrm{T}$ is more likely to be terminated sooner due to bad link quality, thus a higher priority should be assigned in order to decrease the handoff call dropping probability when serving multimedia applications in wireless networks.

B. Packet Success Rate

Before we introduce how to predict the remaining time $\mathrm{T}$ to reach the minimum PSR requirement, it is important to realize the characteristics of PSR. Lal et al. model the packet transmission as a probabilistic process [4]. Suppose k successive packets are transmitted. Let $\mathrm{X}_{\mathrm{i}}$ be a Bernoulli random variable which is 1 if the $i_{\text {th }}$ packet is received successfully, and 0 if not. Assume that $X_{i}$ is independent and identically distributed for $i=$ $1,2, \ldots, k$. The success probability of the random variable $X_{i}$ is equal to PSR. When $\mathrm{k}$ is larger, by the weak law of large numbers, PSR can be closely approximated by $\mathrm{E}\left(\mathrm{X}_{\mathrm{i}}\right)$. Packet success can be defined in several ways. The $\mathrm{PSR}_{\mathrm{s}}$ defined in [4] means that a packet is successful if the first two bytes are correctly received, and if there is at most one single-bit-error in the following bytes. This definition is motivated from the fact that such packets can be recovered by a single-bit-error correction mechanism. Suppose that each packet contains L bytes ( 1 byte $=8$ bits), and then the expected value of $\mathrm{X}_{\mathrm{i}}$ is

$\mathrm{E}\left(\mathrm{x}_{\mathrm{i}}\right)=\left(\left(1-\mathrm{P}_{\mathrm{e}}\right)^{8}\right)^{2}\left(\left(1-\mathrm{P}_{\mathrm{e}}\right)^{8}+8 \mathrm{P}_{\mathrm{e}}\left(1-\mathrm{P}_{\mathrm{e}}\right)^{7}\right)^{\mathrm{L}-2}$

where the $\mathrm{P}_{\mathrm{e}}$ is the bit error probability which depends on the signal-to-noise ratio (SNR) and the modulation scheme. Assume binary FSK with non-coherent detection is used, and the SNR is $\sigma_{\mathrm{i}}$. The bit error probability is

$\mathrm{P}_{\mathrm{e}}=\frac{1}{2} \mathrm{e}^{-\sigma_{\mathrm{i}} / 2}$

Thus (3) can be written as

$\mathrm{E}\left(\mathrm{x}_{\mathrm{i}} \mid \sigma_{\mathrm{i}}\right)=\left(\left(1-\frac{1}{2} \mathrm{e}^{-\sigma_{\mathrm{i}} / 2}\right)^{8}\right)^{2}\left[\left(1-\frac{1}{2} \mathrm{e}^{-\sigma_{\mathrm{i}} / 2}\right)^{7}\left(1+\frac{7}{2} \mathrm{e}^{-\sigma_{\mathrm{i}} / 2}\right)\right]^{\mathrm{L}-2}$

which is a sigmoid as shown in Fig. 3. Here L is assigned as 34 . As Fig. 3 shows, the PSR changes from $0 \%$ to $100 \%$ in a short range of SNR (about $4 \mathrm{~dB}$ to $12 \mathrm{~dB}$ ). There is a knee around 11 $\mathrm{dB}$, and PSR is almost $100 \%$ for more than $11 \mathrm{~dB}$ of SNR. Since the sigmoid is almost linear for most of its range, the expectation of (5), $E_{\sigma i} E\left(X_{i} \mid \sigma_{i}\right)$ can be replaced by $E\left(X_{i} \mid E\left(\sigma_{i}\right)\right)$. Thus the expected value of PSR is
$\mathrm{E}($ PSR $) \approx\left(1-\frac{1}{2} \mathrm{e}^{-\bar{\sigma} / 2}\right)^{16}\left[\left(1-\frac{1}{2} \mathrm{e}^{-\bar{\sigma} / 2}\right)^{7}\left(1+\frac{7}{2} \mathrm{e}^{-\bar{\sigma} / 2}\right)\right]^{32}$

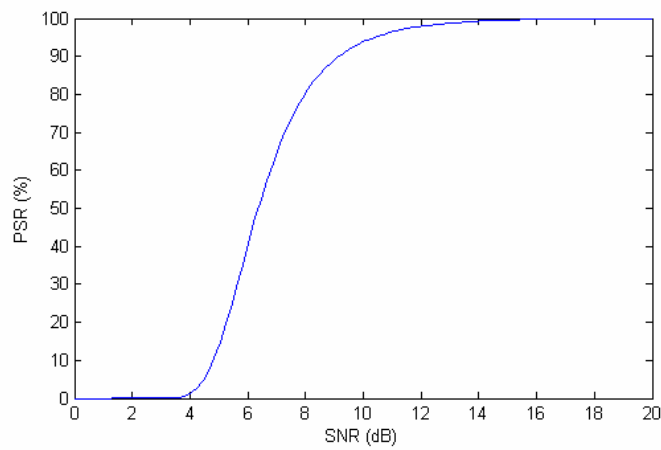

Fig. 3. Expected PSR versus SNR.

From Fig. 3 we can see that, if the minimum PSR requirement of one multimedia service class is $90 \%$, the user would not feel satisfied with the QoS even when the MU gets a $8 \mathrm{~dB}$ SNR because the PSR is only about $80 \%$.

\section{Quality Prediction Priority Queuing}

Assume that there are $\mathrm{m}$ service classes in the wireless communication network, and the minimum PSR requirement for the $i_{\text {th }}$ service class is $\operatorname{PSR}_{\min }(\mathrm{i}), \mathrm{i}=1,2, \ldots, \mathrm{m}$. For the handoff request $\mathrm{j}$ in the handoff queue, its minimum PSR requirement is $\operatorname{PSR}_{\text {min }}\left(C_{j}\right)$, where $C_{j}$ is the service class of $j$. The received PSR of request $j, \operatorname{PSR}_{\text {cur }}(\mathrm{j})$, is successively measured [4]. When $\operatorname{PSR}_{\text {cur }}(\mathrm{j})$ is lower than $\operatorname{PSR}_{\min }\left(\mathrm{C}_{\mathrm{j}}\right)$, the user would feel unsatisfied with the perceived QoS, and the connection would be terminated by him/her. Therefore, higher priority should be assigned to the request $\mathrm{j}$ if $\operatorname{PSR}_{\text {cur }}(\mathrm{j})$ is getting closer and closer to $\operatorname{PSR}_{\min }\left(\mathrm{C}_{\mathrm{j}}\right)$.

We propose a Quality Prediction Priority Queuing (QPPQ) method, which predicts the time remained for the PSR to reach the minimum PSR requirement. Assume the PSR of the handoff request $\mathrm{j}$ is degrading, as Fig. 4 shows. When the received PSR of a call almost reaches its minimum PSR requirement, the relationship between the PSR and the time can be modeled as a linear function in the local region. The PSR of a handoff request is measured and recorded every $\Delta t$ interval. When a handoff request $j$ is at point $A$, the PSR degradation rate $S(j)$, which is the slope of the linear function between the PSR and the time, can be calculated from the current and the previous samples of the PSR, as (7) shows.

$\mathrm{S}(\mathrm{j})=\frac{\operatorname{PSR}_{\text {pre }}(\mathrm{j})-\mathrm{PSR}_{\text {cur }}(\mathrm{j})}{\mathrm{t} 2-\mathrm{t} 1}=\frac{\Delta \operatorname{PSR}(\mathrm{j})}{\Delta \mathrm{t}}$

When the request $\mathrm{j}$ reaches point $\mathrm{B}$, its PSR reaches the minimum requirement, and the call would be terminated by the user. We predict the remaining time for the call to be terminated as (8) shows.

$\mathrm{T}(\mathrm{j})=\frac{\operatorname{PSR}_{\text {cur }}(\mathrm{j})-\mathrm{PSR}_{\min }\left(\mathrm{C}_{\mathrm{j}}\right)}{\mathrm{S}(\mathrm{j})+\alpha}$

where the $\alpha$ is a constant. The purpose of the constant $\alpha$ is to prevent $T(j)$ from becoming infinity when $S(j)$ approaches zero which happens when the MU stops before the traffic light or in the traffic jam or when the current and the previous samples of PSR are the same by chance. It is obvious that higher priority should be assigned to the handoff request $\mathrm{j}$ with lower $\mathrm{T}(\mathrm{j})$ since it is more likely to be terminated soon. 


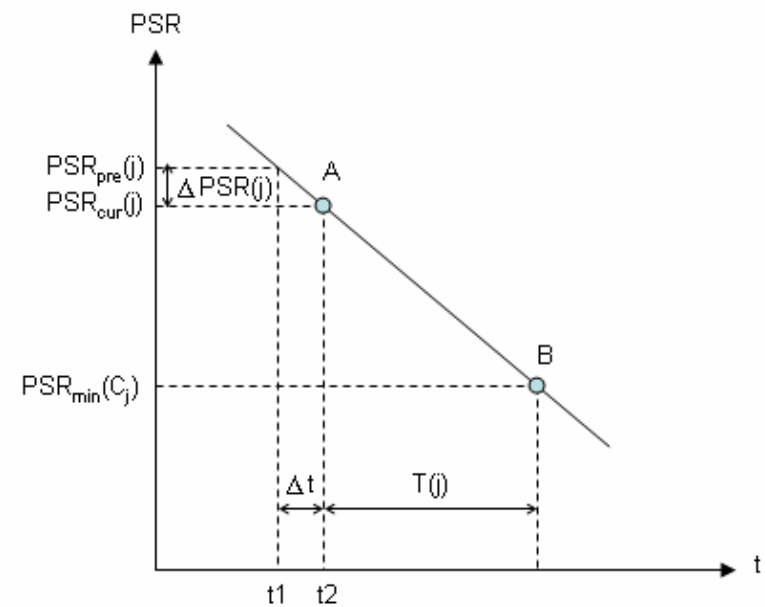

Fig. 4. The prediction of the remaining time for a handoff request $\mathrm{j}$ to reach its minimum PSR requirement.

Please notice that the estimation of $T(j)$ includes some inaccuracy due to the nonlinearity of PSR. Fig. 5 shows the relationship between the PSR and the BS-MU distance. When the MU travels with a constant speed and direction, the physical meaning of the tangent slope at some point on the curve can be regarded as the PSR degradation rate at that point. When a MU is located at point $A$, the estimated $T(j)$ is larger than the real $\mathrm{T}(\mathrm{j})$. However, as the MU keeps on moving towards point $\mathrm{B}$ where the PSR reaches the minimum PSR requirement, the estimation of T(j) would get more and more accurate. Since our method focuses on finding out the handoff request with the highest priority, which is always near the point $\mathrm{PSR}_{\min }$, the inaccuracy of the estimation of $\mathrm{T}(\mathrm{j})$ in fact does not incur any problems.

Therefore, the priority of the handoff request $\mathrm{j}$ in the queue is shown in Eq. (9) .

$$
\mathrm{P}(\mathrm{j})=\frac{1}{\mathrm{~T}(\mathrm{j})}=\frac{\mathrm{S}(\mathrm{j})+\alpha}{\operatorname{PSR}_{\text {cur }}(\mathrm{j})-\mathrm{PSR}_{\text {min }}\left(\mathrm{C}_{\mathrm{j}}\right)}
$$

The handoff request in the queue with the highest priority would be served first when there are channels available in the requested BS.

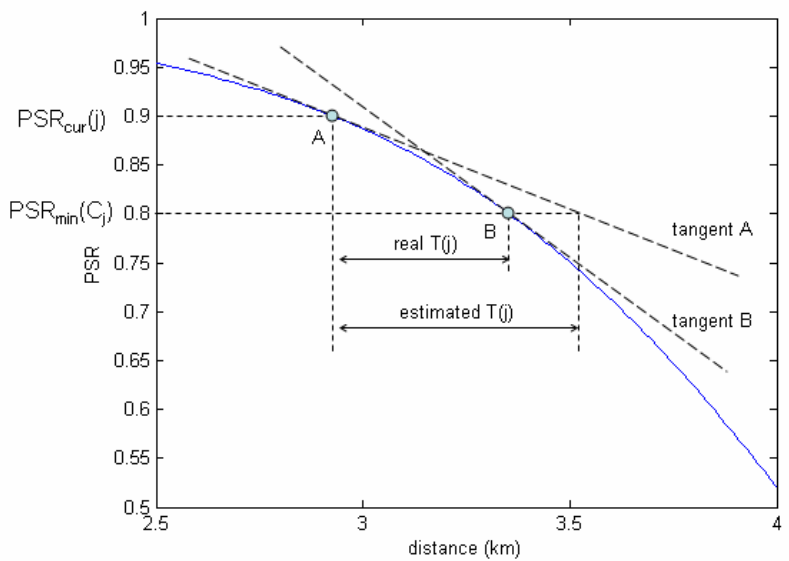

Fig. 5. The difference between the real $\mathrm{T}(\mathrm{j})$ and the estimated $\mathrm{T}(\mathrm{j})$.

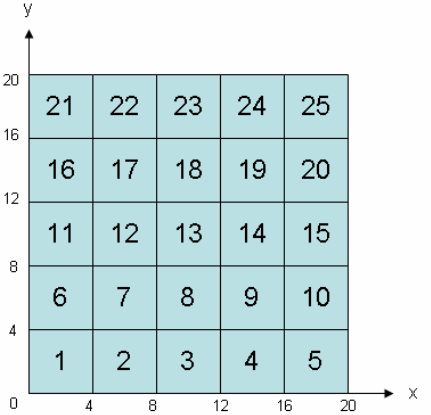

Fig. 6. The simulation environment of the 25 -cell wireless communication network.

\begin{tabular}{|c|c|c|c|}
\hline min. speed & \multicolumn{3}{|c|}{$30 \mathrm{~km} / \mathrm{h}$} \\
\hline max. speed & \multicolumn{3}{|c|}{$90 \mathrm{~km} / \mathrm{h}$} \\
\hline call area & \multicolumn{3}{|c|}{$4 \mathrm{~km} \times 4 \mathrm{~km}$} \\
\hline $\begin{array}{c}\text { channel capacity } \\
\text { per cell }\end{array}$ & \multicolumn{3}{|c|}{$50 \times 64 \mathrm{~kb} / \mathrm{s}$} \\
\hline handoff threshold & \multicolumn{3}{|c|}{$2.83 \mathrm{~km}$} \\
\hline sample interval & \multicolumn{3}{|c|}{1 second } \\
\hline transmit power & \multicolumn{3}{|c|}{$20 \mathrm{~W}$} \\
\hline antenna gain & \multicolumn{3}{|c|}{1} \\
\hline carrier frequency & \multicolumn{3}{|c|}{$900 \mathrm{MHz}$} \\
\hline system loss factor & \multicolumn{3}{|c|}{1} \\
\hline & class 1 & class 2 & class 3 \\
\hline required $\mathrm{BW}$ & $64 \mathrm{~kb} / \mathrm{s}$ & $64 \times 2 \mathrm{~kb} / \mathrm{s}$ & $64 \times 4 \mathrm{~kb} / \mathrm{s}$ \\
\hline mean duration time & $60 \mathrm{~s}$ & $60 \times 5 \mathrm{~s}$ & $60 \times 15 \mathrm{~s}$ \\
\hline required PSR & $80 \%$ & $70 \%$ & $90 \%$ \\
\hline arrival rate ratio & 40 & 10 & 1 \\
\hline
\end{tabular}

Table 2. Simulation parameters.

From Eq. (9), it can be easily seen that the proposed method will assign an earlier handoff to the MU with the worst transmission quality as the denominator shows. Besides, if the MV moves faster, the estimated $S(j)$ will be higher. Hence the handoff priority will also be higher.

\section{SIMULATION DESCRIPTION}

To evaluate the performance of the proposed algorithm, we use the same the simulation environment described in [3]. The simulation environment includes 25 cells as Fig. 6 shows. Each base station is located at the central of each cell with a $4 \times 4 \mathrm{~km}^{2}$ coverage. We assume that the top side and the bottom side of the whole simulation environment are connected together. The same assumption applies to the left side and the right side. It means that when an MU moves up and leaves the simulation environment from cell 23 , it will return to the simulation environment from the bottom side of cell 3 .

In our simulation each new call is generated following the Poisson distribution with the mean arrival rate $\lambda$. We very the value of $\lambda$ to get the corresponding handoff call dropping probability and new call blocking probability. When a new call arrives, its location is randomly chosen in the $20 \times 20 \mathrm{~km}^{2}$ area. The speed of each MU is uniform distributed between 30 and 90 $\mathrm{km} / \mathrm{h}$. Each MU is free to move in any direction.

We design three service classes in the simulation environment. Each service class has its specific bandwidth requirement, mean call duration time, minimum PSR requirement, and arrival rate ratio. Table 2 describes the details of our simulation parameters. 
A static noise level is set as $-67 \mathrm{dBm}$ and we use (2) and (6) to calculate the PSR.

We compare our QPPQ method with the other two methods, including the first-in-first-out (FIFO) and SSMC methods. Each method is simulated for the duration of 24 hours for 10 times. The average handoff call dropping probability and new call blocking probability are used as the metrics to evaluate the performance of various handoff ordering algorithms.

\section{SIMULATION RESULTS}

Fig. 7(a) - (c) show the simulation results of the handoff call dropping probability of the three service classes for the three handoff ordering methods. In the simulation environment, service class 3 requires the highest bandwidth and the highest PSR requirement, and its mean duration time is the longest. Therefore, it is regarded as the most important service class for the operators. Reducing the handoff call dropping probability of service class 3 would bring more revenue to the operators.

When the traffic load is low, every handoff ordering methods perform the same. That is because there are always free channels waiting for handoff requests, and handoff ordering is not necessary. As the traffic load increases, the performance differences between these three methods become obvious. For service class 3, the call dropping probability of QPPQ is reduced approximately about $10 \%$ when compare to that of SSMC. Even in service class 2, QPPQ still performs better than SSMC. For service class 1 QPPQ and SSMC almost perform the same. Generally speaking, QPPQ performs the best for all service classes because it considers the minimum PSR requirements for all service classes and assigns high priority to the handoff request which is reaching its minimum PSR requirement.

Fig. 8(a) - (c) show the simulation results of the new call blocking probability of the three service classes for the three handoff ordering methods. In previous works, reducing the handoff call dropping probability often increases the new call blocking probability. The simulation results show that while reducing the handoff call dropping probability, QPPQ performs almost equal to SSMC about the new call blocking probability.

\section{CONCLUSION}

The objective of the handoff ordering methods for multimedia communications in wireless networks is to reduce the handoff call dropping probability under resource-limited condition. While traditional handoff ordering methods adopt RSS as the basis to prioritize the handoff requests in the queue, we find out that PSR is more suitable for the handoff ordering metric when providing multimedia applications in wireless networks. In this paper we propose a handoff ordering method based on PSR. We predict the remaining time for each $\mathrm{MU}$ to reach its minimum PSR requirement. The priority for each handoff request depends on its remaining time. The major contribution of our method is that it takes care of almost all kinds of service classes available in the multimedia communication networks and reduces the handoff call dropping probability. Simulation results show the proposed algorithm performs the best compared to existing mechanisms.

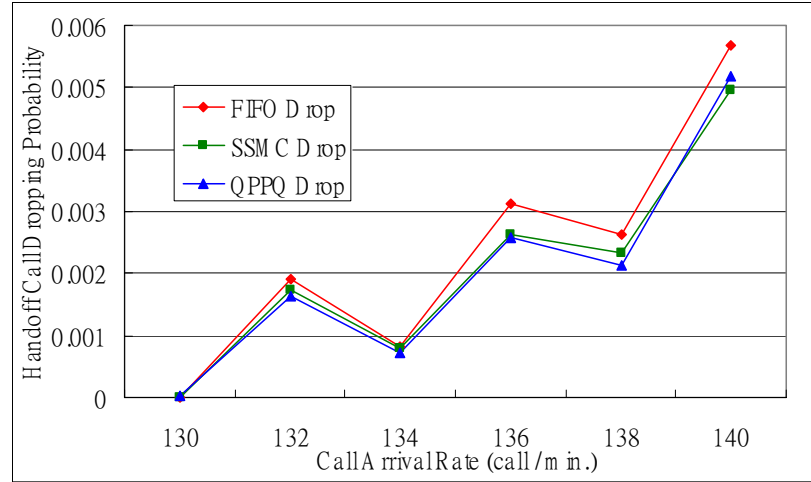

Fig. 7(a). Handoff call dropping probability of class 1 .

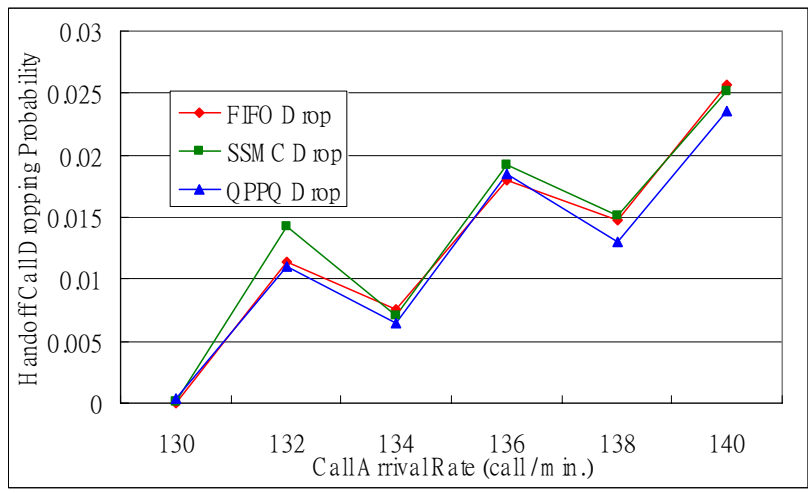

Fig. 7(b). Handoff call dropping probability of class 2 .

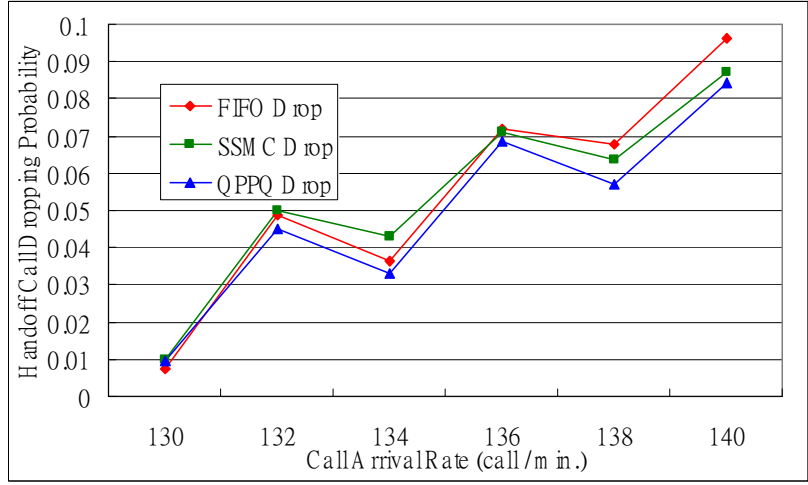

Fig. 7(c). Handoff call dropping probability of class 3 .

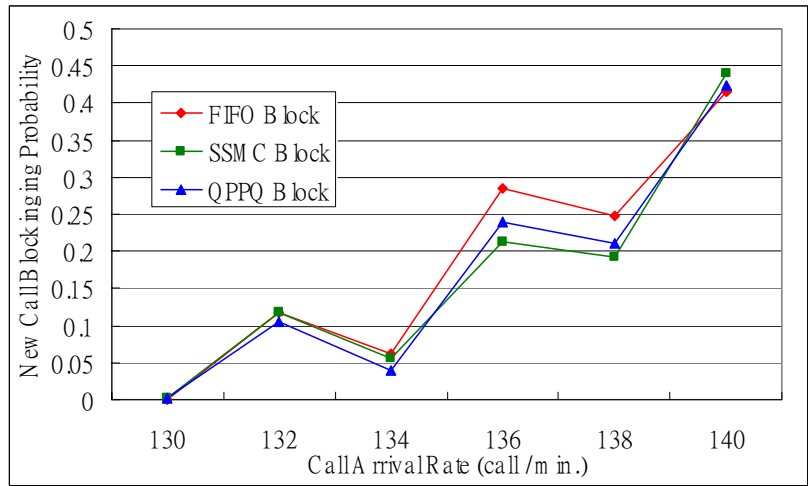

Fig. 8(a). New call blocking probability of class 1 . 


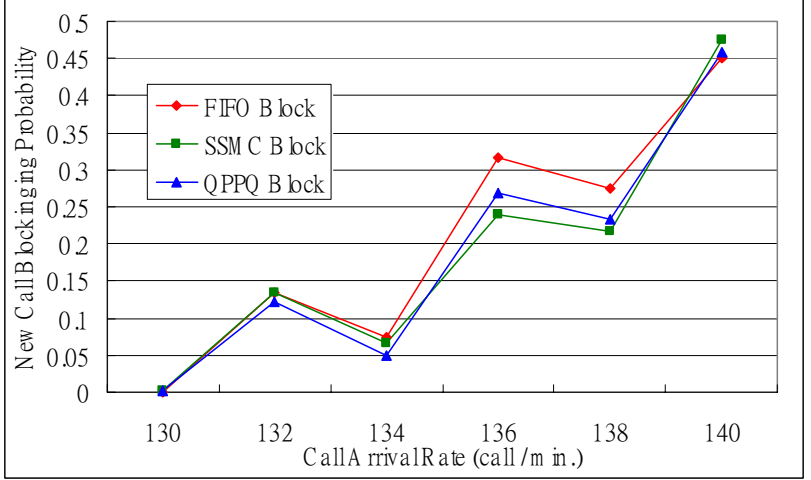

Fig. 8(b). New call blocking probability of class 2 .

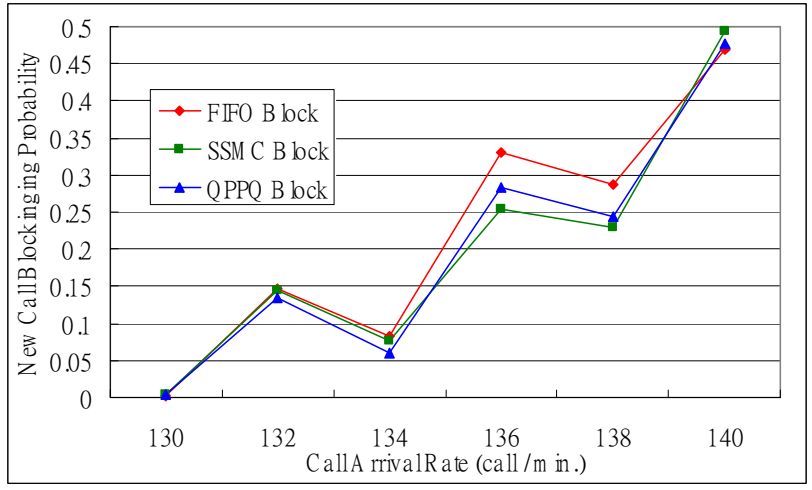

Fig. 8(c). New call blocking probability of class 3 .

\section{REFERENCES}

[1] S. Tekinary and B. Jabbari, "A measurement based prioritization scheme for handovers in cellular and microcellular networks," IEEE J. Select. Areas Commun., pp. 1343-1350, Oct. 1992.

[2] H. G. Ebersman and O. K. Tonguz, "Handoff ordering using signal prediction priority queuing in personal communication system," IEEE Trans. Veh. Technol., vol. 48, pp. 20-35, Jan. 1999.

[3] Ruay-Shiung Chang, Shing-Jiuan Leu, "Handoff Ordering using Signal Strength for Multimedia Communications in Wireless Networks," IEEE Transactions on Wireless Communications, Vol. 3, No. 5, September 2004.

[4] Dhananjay Lal, Arati Manjeshwar, Falk Herrmann, Elif Uysal-Biyikoglu, Abtin Keshavarzian, "Measurement and Characterization of Link Quality Metrics in Energy Constrained Wireless Sensor Networks," GLOBECOM 2003.

[5] Theodore S. Rappaport, "Wireless Communications Principles and Practice," Prentice Hall, 2002, pp. 107-110.

[6] J. Bang, S. Tekinay, and N. Ansari, "Performance analysis of cell switching management scheme in wireless packet communication," in Proc. IEEE GLOBECOM, vol. 6, 2001, pp. 3639-3643.

[7] J. Bang, N. Ansari, and S. Tekinay, "Selective-delay push-in buffering mechanism for QoS provisioning in ATM switching nodes loaded with on-off arrival processes," in Proc. 15th Int. Conf. Information Networking, 2001, pp. 799-804.
[8] J. Bang, S. Tekinay, and N. Ansari, "A novel capacity maximization scheme for multimedia wireless ATM systems providing QoS guarantees for handoffs," in Proc. IEEE 51st Vehicular Technology Conf., vol. 3, 2000, pp. 1747-1751.

[9] Douglas S. J. De Couto, "High-Throughput Routing for Multi-Hop Wireless Networks," PhD thesis, Massachusetts Institute of Technology, 2004. 